\title{
Estafilínidos de la colección de la Sociedad de Ciencias Aranzadi (Coleoptera: Staphylinidae).
}

\section{Rove beetles of the collection of the Society of Sciences Aranzadi (Coleoptera: Staphylinidae).}

Fernando Hiribarnegarai *

$z^{*}$

\section{Resumen}

En el País Vasco y zonas limítrofes aún sigue existiendo una falta de conocimiento de distribución y ocurrencia de especies de estafilínidos. Para paliar parcialmente este desconocimiento, se ha estudiado la colección de la Sociedad de Ciencias Aranzadi. En total se han identificado 102 especies, siendo 46, 32, 6, 3, 8, 6 y 29 nuevas citas para Álava, Bizkaia, Burgos, Cantabria, Gipuzkoa, La Rioja y Navarra respectivamente. Hay también algunos ejemplares procedentes de Asturias, Huesca, Palencia y Ávila. El hecho de que el 90 \% de las especies capturadas haya sido cita nueva para al menos una provincia, demuestra que se debe continuar ampliando el conocimiento a la mayoría de las provincias españolas.

Palabras clave: Staphylinidae, Sociedad de Ciencias Aranzadi, País Vasco, provincias próximas, distribución.

\begin{abstract}
In the Basque Country and neighbouring areas there is still a lack of knowledge concerning the distribution and occurrence of species of rove beetles. In order to partially make up for this lack of knowledge, the collection of rove beetles of the Society of Sciences Aranzadi has been studied. In total, 102 species have been identified, 46, 32, 6, 3, 8, 6 and 29 being new reports for Alava, Biscay, Burgos, Cantabria, Gipuzkoa, La Rioja and Navarre, respectively. There are also a number of specimens from Asturias, Huesca, Palencia and Avila. The fact that $90 \%$ of the captured species were new reports for at least one province, demonstrates that it is necessary to continue extending the knowledge of the family in the majority of Spanish provinces.
\end{abstract}

Key words: Staphylinidae, Aranzadi Sciences Society, Basque Country, neighbouring provinces, distribution. 


\section{Laburpena}

Euskal Herrian eta honen inguruko eremuetan oraindik ere urria da estafilinido espezieen zabalkundeaz eta kokapenenari buruz dagoen ezagutza. Ezagutza urri hori nolabait konpontzeko Aranzadi Natur Zientzien Elkartearen bilduma ikertu da. Denera 102 espezie identifikatu dira, eta horietatik 46, 32, 6, 3, 8, 6 eta 29 zenbakikoak aipamen berriak dira Araba, Bizkaia, Burgos, Kantabria, Gipuzkoa, Errioxa eta Nafarroari dagokienez, hurrenez hurren. Badaude baita espezie batzuk jatorriz Asturias, Huesca, Palencia eta Avilakoak direnak. Atzemandako espezie berrien \%90 aipamen berria izan da gutxienez probintzia baten kasuan eta horrek erakusten du ezagutzan sakontzen jarraitu behar dela, Espainiako probintzia gehienei dagokienean.

Gako hitzak: Staphylinidae, Aranzadi Zientzia Elkartea, Euskal Herria, inguruneko probintziak, hedapena.

\section{r. $\rightarrow$}

\section{Introducción}

La familia Staphylinidae del orden Coleoptera es una de las históricamente más estudiadas en Europa. Con respecto al área tratada en este artículo, es de notar en general la escasa información existente. Trabajos mas actuales van suministrando conocimiento de su distribución como, por ejemplo, los estudios realizados en la bahía de Txingudi de Irún (Anichtchenko, 2006), en el parque natural Aiako Harria (Pagola-Carte, Zabalegi \& Hernando, 2011), en Gipuzkoa (Vorst, 2013) y en zonas del norte de Navarra (Outerelo et al., 2016a, 2016b). También estudios realizados sobre ciencias forenses han proporcionado listados de estafilínidos de Bizkaia y Gipuzkoa (Saloña et al., 2010), (Díaz-Martín, Saloña-Bordas, 2015).

\section{Material y métodos}

El conjunto de la colección Aranzadi se realizó por un grupo de socios entomólogos aficionados y profesionales pertenecientes a la misma entre los años 1964 y 2016. Se ha considerado conveniente su publicación al objeto de ir rellenando los vacíos en el País Vasco y zonas del entorno. En este listado se mencionan principalmente los estafilínidos procedentes de los fondos de entomología de la Sociedad de Ciencias Naturales Aranzadi de San Sebastián y los recogidos por el autor en Álava, Bizkaia y provincias cercanas. La captura de los Staphylinidae se ha realizado sin una metodología precisa en el periodo señalado. Principalmente se utilizó la captura directa y sólo en los tiempos cercanos al año 1995 con trampas Pitfall y de intersección de vuelo. En estos tres últimos años el sistema ha sido, así mismo, la captura directa y las trampas de caída cebadas con materia orgánica en descomposición especialmente en la búsqueda de coleópteros troglófilos. La determinación de la mayoría de los ejemplares capturados por L. Martínez de Murguía en la finca de Artikutza 
en el municipio de Goizueta, (Navarra), fue realizada por R. Outerelo en el año 1995, el resto por el autor del artículo. En general, se han utilizado los tratados clásicos como los de Coiffait (1972), Lompe (2002), Totennham (1954), Tronquet (2014), Portevin (1929), Lott \& Anderson (2011), etc. y trabajos mas específicos como los de Zanetti (2008) y Assing (2010). En el listado general se han simplificado los lugares, fechas de muestreo/captura, legatarios y número de ejemplares según la nota adjunta:

Localidad: Nombre del lugar, (Municipio), Provincia.

Fecha de captura: Día. Mes. Año.

Socios legatarios: $A C=A$. Celada; $C G=C$. Galán; $E C=E$. Cueto; $F G=F$. Garin; $F H=$ F. Hiribarnegarai; JAA = J. A. Albisu; JE = J. Elosegui; LMM = L. Martínez de Murguía.

No. de ejemplares: $1,2,3 \ldots$ ex.

\section{Resultados}

Se estudiaron un total de 102 especies de un total de 416 ejemplares. La especie mas representativa de la colección la constituye Quedius curtipennis (Bernhauer, 1908) con 28 ejemplares seguida de Staphylinus caesareus (Cederhjelm, 1798) con 25, Ocypus olens (O. Müller, 1754) con 22, Quedius mesomelinus (Marsham, 1802) con 22 y Philonthus splendens (Fabricius, 1792) con 15.

Subfamilias

Subfamilia OMALIINAE MacLeay, 1825

Tribu ANTHOPHAGINI Thomson, 1859

Género Lesteva Latreille, 1797

Subgénero Lesteva Latreille, 1797

Lesteva longoelytrata (Goeze, 1777). - Gorbea, (Zeanuri), Bizkaia; 27.04.2010; FH; 12 ex. - Arkamo, (Valdegobia), Álava; 05.05.2016; FH; 1 ex. Primeras citas de Bizkaia y Álava.

Género Olophrum Erichson, 1839

Olophrum piceum (Gyllenhal, 1810). - Laguna de La Cascada, (Neila), Burgos; 03.04.2015; FH; 1 ex. Primera cita para la Península.

Género Philorinum Kraatz, 1858

Philorinum sordidum (Stephens, 1834). - La Arboleda, (Trapagaran), Bizkaia; 15.03.2016; $\mathrm{FH} ; 1$ ex. Primera cita de Bizkaia. 
Tribu EUSPHALERINI Hatch, 1957

Género Eusphalerum Kraatz, 1857

Subgénero Eusphalerum Kraatz, 1857

Eusphalerum anale (Erichson, 1840). - Artikutza, (Goizueta), Navarra; 13.11.1995; LMM; 3 ex. Primera cita de Navarra.

Tribu OMALIINI MacLeay, 1825

Genero Omalium Gravenhorst, 1802

Omalium allardii (Fairmaire \& Brisout, 1859). - Gorbea, (Zuia), Álava; 27.04.2010; FH; 2 ex. Primera cita de Álava.

Omalium rivulare (Paykull, 1789). - Betarres, (Medina de Pomar), Burgos; 19.10.2016; $\mathrm{FH} ; 1$ ex.

Género Paraphloeostiba Steel, 1960

Paraphloeostiba gayndahensis (MacLeay, 1873). - Elordui, (Erandio), Bizkaia; 25.01.2016; $\mathrm{FH} ; 10$ ex. Primera cita de Bizkaia.

Subfamilia PROTEININAE Erichson, 1839

Tribu PROTEININI Erichson, 1839

Genero Proteinus Latreille, 1797

Proteinus brachypterus (Fabricius, 1792). - Betarres, (Medina de Pomar), Burgos; 07.10.2016; $\mathrm{FH} ; 2$ ex. Primera cita de Burgos.

Subfamilia TACHYPORINAE MacLeay, 1825

Tribu MYCETOPORINI Thomson, 1859

Género Bolitobius Leach, 1819

Subgénero Bolitobius Leach, 1819

Bolitobius castaneus (Stephens, 1832). - Arangoiti, (Romanzado), Navarra; 23.06.1977; JAA; 1 ex. Primera cita de Navarra.

Género Lordithon Thomson, 1859

Subgénero Lordithon Thomson, 1859

Lordithon thoracicus (Fabricius, 1777). - Urbina Basabekoa, (Kuartango), Alava; 30.11.2003; $\mathrm{FH} ; 2$ ex. Primera cita de Álava. 
Género Mycetoporus Manerheim, 1830

Mycetoporus longulus (Mannerheim, 1830). - Gorbea, (Zuia), Álava; 01.06.2010; FH; 2 ex.

Primera cita de Álava.

Género Sepedophilus Gistel, 1856

Sepedophilus marshami (Stephens, 1832). - Urbina Basabekoa, (Kuartango), Álava;

29.03.2003; FH; 1 ex. - La Llana, (Zuia), Álava; 21.04.2012; FH; 1 ex. Primera cita de Álava.

Género Tachinus Gravenhorst, 1802

Subgénero Tachinus Gravenhorst, 1802

Tachinus flavolimbatus (Pandellé, 1869). - Ondiz, (Leioa), Bizkaia; 20.04.1994; FH; 1 ex.

Primera cita de Bizkaia.

Tachinus humeralis (Gravenhorst, 1802). - Urbina Basabekoa, (Kuartango), Álava; 08.10.2000; FH; 4 ex. - Pagazuri, (Zigoitia), Álava; 22.06.2016; FH; 3 ex. Primera cita de Álava. Segunda cita concreta de la Península.

Tachinus rufipes (De Geer, 1774). - Ulia, (Donostia), Gipuzkoa; 24.03.1982; FG; 1 ex. - Jaizkibel, (Hondarribia), Gipuzkoa; 02.04.1982; FG; 1 ex. - Aralar, (Uharte-Arakil), Navarra; 15.05.1982; JAA; 1 ex. - Urbina Basabekoa, (Kuartango), Álava; 15.08.1999; FH; 2 ex. - Universidad, (Leioa), Bizkaia; 17.06.2005; FH; 1 ex. - Pico Mayor, (Galdames), Bizkaia; 26.04.2016; FH; 1 ex. Primeras citas de Álava, Bizkaia y Navarra.

Género Tachyporus Gravenhorst, 1802

Subgénero Palporus Campbell, 1979

Tachyporus nitidulus (Fabricius, 1781). - Nabatzua, (Valdegobia), Álava; 09.07.2016; FH; 1 ex. Primera cita de Álava.

\section{Subgénero Tachyporus Gravenhorst, 1802}

Tachyporus atriceps (Stephens, 1832). - Marinda, (Kuartango), Álava; 21.02.2016; FH; 1ex. Primera cita de Álava.

Tachyporus chrysomelinus (Linnaeus, 1758). - Eretza, (Gueñes), Bizkaia; 10.08.1986; FH; 1 ex. Primera cita de Bizkaia.

Tachyporus hypnorum (Fabricius, 1775). - Ondiz, (Leioa), Bizkaia; 07.05.1992; FH; 2 ex.

Primera cita de Bizkaia.

Tachyporus tersus (Erichson, 1839). - Pagazuri, (Zigoitia), Alava; 21.03.2009; FH; 1 ex.

Primera cita de Álava. Amplía la distribución conocida en la Península 
Subfamilia ALEOCHARINAE Fleming, 1821

Tribu ALEOCHARINI Fleming, 1821

Género Aleochara Gravenhorst, 1802

Subgénero Aleochara Gravenhorst, 1802

Aleochara curtula (Goeze, 1777). Mendizorrotz, (Donostia), Gipuzkoa; 20.04.1982; FG; 1 ex. - Subijana, (Ribera Alta), Álava; 14.07.1982; EC; 12 ex. - Jaizkibel, (Hondarribia), Gipuzkoa; 21.08.1982; JAA; 1 ex. - Urbina Basabekoa, (Kuartango), Álava; 10.06.2005; FH; 2 ex. Primera cita de Álava.

Aleochara intricata (Mannerheim, 1830). - Lando, (Romanzado), Navarra; 25.07.1982; JAA; 4 ex. - Artikutza, (Goizueta), Navarra; 24.07-07.08.1995; LMM; 1 ex. - Urbina Basabekoa, (Kuartango), Álava; 06.07.2001; FH; 1 ex. - Hayal de Guinea, (Valdegobia), Alava; 10.09.2016; $\mathrm{FH} ; 1$ ex. Primeras citas de Álava y Navarra.

Subgénero Coprochara Mulsant \& Rey, 1873

Aleochara bipustulata (Linnaeus, 1761). - Arraiotz, (Baztan), Navarra; 17.07.1982; FG; 1 ex. Gorbea, (Zeanuri), Bizkaia; 22.06.2013; FH; 1 ex. - Oiz mendia, (Berriz), Bizkaia; 29.06.2016; FH; 6 ex. - Nabatzua, (Valdegobia), Álava; 09.07.2016; FH; 5 ex. Primeras citas de Álava, Bizkaia y Navarra.

Subgénero Xenochara Mulsant \& Rey, 1873

Aleochara lanuginosa (Gravenhorst, 1802). - Aralar, (Uharte-Arakil), Navarra; 15.08.1982; JAA; 2 ex. - Hayal de Guinea, (Valdegobia), Álava; 10.09.2016; FH; 1 ex. Primeras citas de Navarra y Álava.

Aleochara tristis (Gravenhorst, 1806). - Urramuta, (Kuartango), Álava; 26.04.2015; FH; 2 ex. - Uraiaga, (Galdames), Bizkaia; 29.04.2016; FH; 1 ex. - Pagazuri, (Zigoitia), Álava; 28.07.2016; FH; 1 ex. - Punta Lucero, (Zierbena), Bizkaia; 08.09.2016; FH; 1 ex. - Arriano, (Kuartango), Álava; 10.09.2016; FH; 1 ex. Primeras citas de Álava y Bizkaia.

Tribu AUTALIINI Thomsom, 1859

Género Autalia LEACH, 1819

Autalia impressa (Olivier, 1765). - Betarres, (Medina de Pomar), Burgos; 07.10.2016; FH; 1 ex. Primera cita de Burgos.

Tribu HOMALOTINI Heer, 1839

Género Bolitochara Mannerheim, 1830

Subgénero Ditropalia Casey, 1906

Bolitochara obliqua (Erichson, 1837). - (Santa Coloma), La Rioja; 30.10.1982; EC; 2 ex. Primera cita de La Rioja. 
Tribu LOMECHUSINI Fleming, 1821

Género Pella Stephens, 1835

Subgénero Pella Stephens, 1835

Pella limbata (Paykull, 1789). - Gorbea, (Zeanuri), Bizkaia; 01.06.2010; FH; 1 ex. Primera cita de Bizkaia.

Subfamilia OXYTELINAE Fleming, 1821

Tribu OXYTELINI Fleming, 1821

Género Anotylus Thomson, 1859

Anotylus inustus (Gravenhorst, 1806). - Alto de Coronas (Romanzado), Navarra; 28.05.1978; JAA; 1 ex. - Arangoiti, (Romanzado), Navarra; 02.05.1982; JAA; 1 ex.

Anotylus plagiatus (Rosenhauer, 1856). - Artikutza, (Goizueta), Navarra; 18.06.1995; LMM; 1 ex. Primera cita de Navarra.

Género Oxytelus Gravenhorst, 1802

Subgénero Oxytelus Gravenhorst, 1802

Oxytelus piceus (Linnaeus, 1767). - Lando, (Romanzado), Navarra; 25.07.1982; JAA; 2 ex.

Subgénero Tanycraerus Thomson, 1859

Oxytelus laqueatus (Marsham, 1802). - Urbina Basabekoa, (Kuartango), Álava; 06.08.2000; FH; 2 ex. - Aralar, (Uharte-Arakil), Navarra; 19.07.2016; FH; 2 ex. Primeras citas de Álava y Navarra.

Género Platystethus Mannerheim, 1830

Subgénero Craetopycrus Tottenham, 1939

Platystethus cornutus (Gravenhorst, 1802). - Nabatzua, (Valdegobia), Álava; 09.07.2016; FH; 1 ex. - Lanunze, (Kuartango), Álava; 07.08.2016; FH; 1 ex. Primera cita de Álava.

Platystethus spinosus (Erichson, 1840). - Hayedo de Lizarrusti, (Arbizu), Navarra; 18.06.1982; FG; 1 ex. - Betarres, (Medina de Pomar), Burgos; 06.05.2007; FH; 1 ex. - Gorbea, (Zeanuri), Bizkaia; 22.06.2011; FH; 1 ex. - Nabatzua, (Valdegobia), Álava; 10.10.2015; FH; 1 ex. - La Arboleda, (Trapagaran), Bizkaia; 15.03.2016; FH; 1 ex. Primeras citas de Álava, Bizkaia y Burgos.

\section{Subgénero Platystethus s.str.}

Platystethus arenarius (Geoffroy, 1785). - (Donostia), Gipuzkoa; 24.07.1982; JAA; 10 ex. Lando, (Romanzado), Navarra; 25.07.1982; JAA; 1 ex. - Korretunde, (Kuartango), Álava; 25.08.2013; FH; 1 ex. - Punta Lucero, (Zierbena), Bizkaia; 08.09.2016; FH; 1 ex. Primeras citas de Álava y Navarra. 
Género Bledius Leach, 1819

Subgénero Bledius Leach, 1819

Bledius unicornis (Germar, 1825). - Río Butrón, (Plentzia), Bizkaia; 21.07.2015; FH; 1 ex.

Primera cita de Bizkaia.

Género Planeustomus Jaquelin du Val, 1857

Planeustomus kahrii (Kraatz, 1857). - Korretunde, (Kuartango), Álava; 25.08.2013; FH; 1 ex.

Primera cita de Álava.

Subfamilia STENINAE MacLeay, 1825

Género Stenus Latreille, 1797

Subgénero Hemistenus Motschoulsky, 1860

Stenus ochropus (Kiessenwetter, 1858). - Artikutza, (Goizueta), Navarra; 07.05.1995; LMM; 1 ex. - Betarres, (Medina de Pomar), Burgos; 07.10.2016; FH; 1 ex. Primeras citas de Navarra y Burgos. Amplía su distribución a la mitad norte de la Península.

Subgénero Stenus Latreille, 1797

Stenus ocellatus (Fauvel, 1865). - Río Urumea, (Goizueta), Navarra; 02.06.1982; FG; 1 ex.

Primera cita de Navarra.

Subfamilia PAEDERINAE Fleming, 1821

Tribu PAEDERINI Fleming, 1821

Género Achenium Leach, 1819

Subgénero Achenium Leach, 1819

Achenium depressum (Gravenhorst, 1802). - Nabatzua, (Valdegobia), Álava; 10.10.2015; FH; 2 ex. Primera cita de Álava.

Género Lobrathium Mulsant \& Rey, 1878

Subgénero Lobrathium Mulsant \& Rey, 1878

Lobrathium multipunctum (Gravenhorst, 1802). - Otsondo, (Baztan), Navarra; 09.04.1983; FG; 1 ex. - Gorbea, (Zeanuri), Bizkaia; 05.05.2014; FH; 1 ex. Primera cita de Bizkaia.

Género Tetartopeus Czwalina, 1888

Tetartopeus angustatus (Lacordaire, 1835). - Gorbea, (Zuia), Álava; 02.06.2009, FH; 1 ex. Primera cita de Álava. 
Género Paederidus Mulsant \& Rey, 1878

Paederidus ruficollis (Fabricius, 1777). - Urumea, (Goizueta), Navarra; 02.06.1982; FG; 5 ex. (San Millán), La Rioja; 17.04.1982; EC; 1 ex. - (Puente Viesgo), Cantabria; 13.05.1982; 9 ex. Embalse de Urkulu, (Aretxabaleta), Gipuzkoa; 17.05.2016; FH; 1 ex. Primeras citas de Cantabria y La Rioja.

Género Paederus, Fabricius, 1775

Subgénero Eopaederus, Scheerpeltz, 1957

Paederus caligatus (Erichson, 1840). - Montes de Izki, (Korres), Álava; 27.05.1997; FH; 1 ex. Primera cita de Álava.

Subgénero Heteropaederus Scheerpeltz, 1957

Paederus fuscipes (Curtis, 1826). - Regolfo, (Solórzano), Cantabria; 17.05.2014; FH; 1 ex. - Agiturri, (Kuartango), Álava; 06.07.2014; FH; 1 ex.

Subgénero Paederus Fabricius, 1775

Paederus riparius (Linnaeus, 1758). - Embalse de Urkulu, (Aretxabaleta), Gipuzkoa; 17.05.2016; FH; 1 ex. Primera cita de Gipuzkoa. Amplía su distribución a la mitad norte de la Península.

Subgénero Poederomorphus Gautier, 1862

Paederus littoralis (Gravenhorst, 1802). - Mendizorrotz, (Donostia), Gipuzkoa; 20.05.1977; JAA; 1 ex. - Mendizorrotz, (Donostia), Gipuzkoa; 20.05.1982; FG. 1 ex. - Ondiz, (Leioa), Bizkaia; 26.04.1994; FH; 1 ex. - Urbina Basabekoa, (Kuartango), Álava; 20.05.2001; FH; 1 ex. - Regolfo, (Solórzano), Cantabria; 17.05.2014; FH; 2 ex. Primera cita de Bizkaia.

Subfamilia STAPHYLININAE Latreille, 1802

Tribu OTHIINI Thomson, 1859

Género Atrecus Jaqueline du Val, 1856

Atrecus affinis (Paykull, 1798). - Saldropo, (Zeanuri), Bizkaia; 28.03.1999; FH; 1 ex.

Primera cita de Bizkaia.

Género Othius Stephens, 1832

Othius punctulatus (Goeze, 1777). - Artikutza, (Goizueta), Navarra; 24.07.1995; LMM; 2 ex. Primera cita de Navarra. 
Tribu STAPHYLININI Latreille, 1802

Subtribu PHILONTHINA Kirby, 1837

Género Bisnius Stephens, 1829

Bisnius fimetarius (Gravenhorst, 1802). - Pagazuri, (Zigoitia), Álava; 22.06.2016; FH; 3 ex. -Aralar, (Uharte-Arakil), Navarra; 19.07.2016; FH; 1 ex. Primera cita de Álava.

Bisnius sordidus (Gravenhorst, 1802). -Lupetzeta, (Orozko), Bizkaia; 25.08.1991; FH; 1 ex. - Igitaia, (Zeanuri), Bizkaia; 27.05.2015; FH; 1 ex. Primera cita de Bizkaia.

\section{Género Erichsonius Fauvel, 1874}

Subgénero Stectophilonthus Tottenham, 1949

Erichsonius signaticornis (Mulsant \& Rey, 1853). - Gorbea, (Zuia), Álava; 02.06.2009; FH; 1 ex. - Embalse de Urkulu, (Aretxabaleta), Gipuzkoa; 17.05.2016; FH; 1 ex. Primera cita de Álava.

\section{Género Gabrius Curtis, 1829}

Gabrius nigritulus (Gravenhorst, 1802). - La Llana, (Zuia), Álava; 21.04.2012; FH; 1 ex. - Gorbea, (Zeanuri), Bizkaia; 14.04.2016; FH; 1 ex. Primeras citas de Álava y Bizkaia.

Gabrius splendidulus (Gravenhorst, 1802). - Ibarla, (Irun), Gipuzkoa; 10.04.1982; EC; 1 ex. Primera cita de Gipuzkoa.

Gabrius tirolensis (Luze, 1903). - Artikutza, (Goizueta), Navarra; 07.05.1997; LMM; 1 ex.

Primera cita de Navarra.

Género Neobisnius Ganglbauer, 1895

Neobisnius procerulus (Gravenhorst, 1806). - Lanunze, (Kuartango), Álava; 26.07.2015;

FH; 1 ex. Primera cita de Álava.

Género Philonthus Stephens, 1829

Subgénero Onychophilonthus Nereisheimer \& Wagner, 1924

Philonthus marginatus (Müller, 1764). - Betzulako Lepoa, (Otsagabia), Navarra; 26.08.2016; $\mathrm{FH} ; 1$ ex. Primera cita de Navarra.

Subgénero Philonthus Stephens, 1829

Philonthus cognatus (Stephens, 1832). - Bisaurin, (Aragüés del Puerto), Huesca; 27.05.1978; JAA; 1 ex. - Ondiz, (Leioa), Bizkaia; 18.03.1992; FH; 3 ex. Primera cita de Bizkaia.

Philonthus concinnus (Gravenhorst, 1802). - Lando, (Romanzado), Navarra; 25.07.1982; JAA; 8 ex. - Gorbea, (Zeanuri), Bizkaia; 28.07.2016; FH. 1 ex. Primeras citas de Bizkaia y Navarra. 
Philonthus cruentatus (Gmelin, 1790). - Lando, (Romanzado), Navarra; 25.07.1982; JAA; 15 ex. - Sierra de Gibixo, (Kuartango), Álava; 06.08.2000; FH; 1 ex. Primeras citas de Álava y Navarra.

Philonthus ebeninus (Gravenhorst, 1802). - Urbasa, (Olazagutia), Navarra; 26.06.1982; JAA; 1ex.

Philonthus laminatus (Creutzer, 1799). - Aralar, (Uharte-Arakil), Navarra; 25.05.1982; JAA; 1 ex. Primera cita de Navarra.

Philonthus politus (Linnaeus, 1758). - Mendizorrotz, (Donostia), Gipuzkoa; 23.05.1982; JAA; 1 ex.

Philonthus sanguinolentus (Gravenhorst, 1802). - (Donostia), Gipuzkoa; 24.08.1982; JAA; 4 ex.

Philonthus splendens (Fabricius, 1792). - Bisaurin, (Aragüés del Puerto), Huesca; 27.05.1978; JAA; 1 ex. - (San Millán), La Rioja; 17.04.1982; EC; 2 ex. - Mendizorrotz, (Donostia), Gipuzkoa; 23.05.1982; JAA; 1 ex. - Lando, (Romanzado), Navarra; 25.07.1982; JAA; 9 ex. - Ondiz, (Leioa), Bizkaia; 07.05.1992; FH; 1 ex. - Urbina Basabekoa, (Kuartango), Álava; 01.08.1999; F.H; 1 ex. Primeras citas de Álava, Bizkaia y Huesca.

Philonthus succicola (Thomson, 1860). - Lupetzeta, (Orozko), Bizkaia; 25.08.1991; FH; 1 ex. - Regolfo, (Solorzano), Cantabria; 27.05.2014; FH; 1 ex. Primeras citas de Bizkaia y Cantabria.

Philonthus varians (Paykull, 1789). - Aralar, (Uharte-Arakil), Navarra; 15.08.1982; JAA; 2 ex. Primera cita de Navarra.

Género Quedius Stephens, 1829

Subgénero Distichalius Casey, 1915

Quedius cinctus (Paykull, 1790). - Collado de la Centenera, (El Arenal), Ávila; 02.04.1983; FG; 1 ex. - Urbina Basabekoa, (Kuartango), Álava; 10.06.2005; FH; 1 ex. Primera cita de Álava.

Subgénero Microsaurus Dejean, 1833

Quedius fulgidus (Fabricius, 1793). - Ondarreta, (Donostia), Gipuzkoa; 04.06.1982; FG; 1 ex. Primera cita de Gipuzkoa.

Quedius mesomelinus (Marsham, 1802). - Cueva de Alli, (Larraun), Navarra; 05.11.1967; CG; 14 ex. - Cueva de Aitzbitarte III, (Errenteria), Gipuzkoa; 12.03.1968; AC; 1 ex. - Cueva de Repiko, (Valdegobia), Álava; 17.09.2014; FH; 7 ex. Primera cita de Álava.

Quedius ochripennis (Menetriès, 1832). - Nuestra Sra. de Alconada, (Ampudia), Palencia; 25.08.2008; FH; 1 ex. Primera cita de Palencia. 
Subgénero Quedius Stephens, 1829

Quedius curtipennis (Bernhauer, 1908). - (Roncal), Navarra; 25.08.1978; JAA; 1 ex. - Otzaurte, (Zegama), Gipuzkoa; 01.05.1982; FG; 1 ex. - (San Millán), La Rioja; 17.04.1982; - Urkiola, (Abadiño), Bizkaia; 02.04.1992; FH; 1 ex. - Artikutza, (Goizueta), Navarra; 1995 a 1997; LMM; 23 ex. Primeras citas de Bizkaia, Gipuzkoa, Navarra y La Rioja.

Quedius fuliginosus (Gravenhorst, 1802). - Artikutza, (Goizueta), Navarra; 08.01.1996; LMM; 1 ex. Primera cita de Navarra.

Quedius levicollis (Brullé, 1832). - Jaizkibel, (Hodarribia), Gipuzkoa; 12.05.1982; EC; 1 ex. Primera cita de Gipuzkoa.

Quedius molochinus (Gravenhorst, 1806). - Lobera, (Kuartango), Álava; 20.12.2014; FH; 1. Primera cita de Álava.

Subgénero Raphirus Stephens, 1829

Quedius boops (Gravenhorst, 1802). - Gorbea, (Zuia), Álava; 07.05.2009; FH; 2 ex. - Puerto de Piqueras, (Lumbreras), La Rioja; 09.01.2013; FH; 1 ex. Primeras citas de Álava y La Rioja.

Quedius humeralis (Stephens, 1832). - Artikutza, (Goizueta), Navarra; 19.01.1997; LMM; 2 ex. Primera cita de Navarra.

Quedius lateralis (Gravenhorst, 1802). - (San Millán), La Rioja; 04.05.1982; EC; 1 ex. - Ondiz, (Leioa), Bizkaia; 1993; FH; 1 ex. Primeras citas de La Rioja y Bizkaia.

Género Creophilus Leach, 1819

Creophilus maxillosus (Linnaeus, 1758). - Jaizkibel, (Hondarribia), Gipuzkoa; 24.08.1982; EC; 1 ex. - Lupetzeta, (Orozko), Bizkaia; 1992; FH; 1 ex. - Santa Eulalia, (Kuartango), Álava; 07.08.2016; FH; 1 ex. Primeras citas de Álava y Bizkaia.

Género Dinothenarus Thomson, 1858

Subgénero Dinothenarus Thomson, 1858

Dinothenarus pubescens (De Geer, 1774). - Urbina Basabekoa, (Kuartango), Álava; 15.06.2003; FH; 1 ex. Primera cita de Álava.

Género Emus Leach, 1819

Emus hirtus (Linnaeus, 1758). - Urbasa, (Olazagutia), Navarra; 24.07.1978; JAA; 2 ex. - La Alta, (Galdames), Bizkaia; 10.08.1991; FH; 1 ex. - Urbina Basabekoa, (Kuartango), Álava; 20.05.2001; FH; 1 ex. - Gorbea, (Zuia), Álava; 04.06.2012; FH; 1 ex. Primeras citas de Álava, Bizkaia y Navarra. 
Género Ocypus Leach, 1819

Subgénero Matidus Motschulsky, 1860

Ocypus pedemontanus (G.Müller, 1924), ssp. cantabricus (G.Müller, 1926). - Pineta, (Bielsa), Huesca; 04.08.1982; FG; 1 ex. - Lizarrusti, (Etxarri-Aranaz), Navarra; 18.06.1982; FG; 3 ex. - Aralar, (Uharte-Arakil), Navarra; 15.08.1982; JAA; 1 ex. - Puerto de Bidania, (Bidegoien), Gipuzkoa; 21.05.1982; FG; 2 ex. - Korretunde, (Kuartango), Álava; 18.09.2005; FH; 2 ex. Primeras citas de Gipuzkoa y Navarra.

Ocypus olens (O. Müller, 1754). - (Aia), Gipuzkoa; 21.07.1963; JE; 1 ex. Azkarate, (Araitz), Navarra; 19.09.1964; JE; 1 ex. - Ategorrieta, (Donostia), Gipuzkoa; 03.11.1967; JE. 1 ex. - Arangoiti, (Romanzado), Navarra; 04.07.1982; JAA; 1 ex. - San Miguel de Aralar, (Uharte-Arakil), Navarra; 21.08.1982; FG; 7 ex. - Lizarraga, (Ergoiena), Navarra; 26.06.1982; JAA; 1 ex. - Fadura, (Getxo), Bizkaia; 05.10.1983; FH; 2 ex. - Artikutza, (Goizueta), Navarra; 21.08-04.09.1995; LMM; 7 ex. - Arangatxa, (Kuartango), Álava; 21.08.2005; FH; 1 ex. Primeras citas de Bizkaia y Navarra.

Ocypus ophtalmicus (Scopoli, 1763), ssp. atrocyaneus (Fairmaire, 1860). - Ondiz, (Leioa), Bizkaia; 16.03.1993; FH; 1 ex. - Arroyo San Juan, (Lara de Los Infantes), Burgos; 16.10.2005; FH; 1 ex. - Marinda, (Kuartango), Álava; 07.10.2007; FH; 1 ex. - Lutxana, (Erandio), Bizkaia; 13.10.2015; FH; 1 ex. Primeras citas de Álava y Bizkaia.

Subgénero Pseudocypus Mulsant \& Rey, 1876

Ocypus aeneocephalus (De Geer, 1774). - Gorbeagane, (Zeanuri), Bizkaia; 14.04.2016; FH; 1 ex. - Montenegro, (Viniegra de Arriba), La Rioja; 18.04.2014; FH; 1 ex. Primeras citas de Bizkaia y La Rioja.

Ocypus aethiops (Waltl, 1835). - Leire, (Yesa), Navarra; 26.07.1977; JAA; 3 ex. Primera cita de Navarra.

Ocypus obscuroaeneus (Fairmaire, 1852). - Zarate, (Zuia), Álava; 13.04.2010; FH; 2 ex. Primera cita de Álava .

Género Ontholestes Ganglbauer, 1895

Ontholestes murinus (Linnaeus, 1758). - (Donostia), Gipuzkoa; 24.08.1982; JAA; 2 ex. - Jaizkibel, (Hondarribia), Gipuzkoa; 24.08.1982; EC; 1 ex. - Bisaurin, (Aragüés del Puerto), Huesca; 27.05.1978; JAA; 2 ex. - Entzia, (Andoin), Álava; 08.05.1982; JAA; 1 ex. - (San Millán), La Rioja; 04.05.1982; EC; 1 ex. - Lando, (Romanzado), Navarra; 25.07.1982; JAA; 1 ex. - Lupetzeta, (Orozko), Bizkaia; 26.04.1992; FH; 1 ex. - Urbina Basabekoa, (Kuartango), Álava; 20.05.2001; FH; 1 ex. - Barriga, (Valle de Losa), Burgos; 17.07.2005; FH; 2 ex. Primeras citas de Álava, Bizkaia, Gipuzkoa y Burgos.

Ontholestes tessellatus (Geoffroy, 1785). - Urbina Basabekoa, (Kuartango), Álava; 10.06.2005; FH; 1 ex. - Regolfo, (Solorzano), Cantabria; 17.05.2014; FH; 3 ex. Primeras citas de Álava y Cantabria. 
Género Platydracus Thomson, 1858

Platydracus flavopunctatus (Latreille, 1804). - Urbina Basabekoa, (Kuartango), Álava; 20.08.2000; FH; 1 ex. Primera cita de Álava.

Platydracus fulvipes (Scopoli, 1763). - Lagos de Covadonga, (Cangas de Onís), Asturias; 12.05.1982; JAA; 1 ex.

Platydracus meridionalis (Rosenhauer, 1847). - Amaiur, (Baztan), Navarra; 18.07.1982; JAA; 1 ex. - Subijana, (Ribera Alta), Álava; 14.07.1982; EC; 1 ex. Primeras citas de Álava y Navarra.

Género Staphylinus Linnaeus, 1758

Staphylinus caesareus (Cederhjelm, 1798). - Mendizorrotz, (Donostia), Gipuzkoa; 01.06.1977; JAA; 3 ex. - Belate, (Baztan), Navarra; 13.04.1980; JAA; 14 ex. - Jaizkibel, (Hondarribia), Gipuzkoa; 24.05.1982; EC; 2 ex. - Otzaurte, (Zegama), Gipuzkoa; 01.05.1982; FG; 1 ex. - Gorbea, (Zeanuri), Bizkaia; 23.06.1984; FH; 2 ex. - Bosque de Irati, (Otsagabia), Navarra; 22.08.1990; FH; 1 ex. - Peñas de Aya, (Oiartzun), Gipuzkoa; 12.06.1991; F.H; 1 ex. - UrkuluOlaldea, (Aezkoa), Navarra; 09.04.1993, FH; 1 ex. Primeras citas de Navarra y Bizkaia.

Género Tasgius Stephens, 1829

Subgénero Rayacheila Motschulsky, 1845

Tasgius morsitans (Rossi, 1790). - Artikutza, (Goizueta), Navarra; 29.08.1994; LMM; 1 ex.

Primera cita de Navarra.

Tasgius winkleri (Bernhauer, 1906). - Arroyo Iturrieta, (Kuartango), Álava; 15.04.2004; FH; 2 ex. Primera cita de Álava.

Tribu XANTOLININI Erichson, 1839

Género Gyrohypnus Leach, 1819

Subgénero Gyrohypnus Leach, 1819

Gyrohipnus fracticornis (O. Müller, 1776). - (Donostia), Gipuzkoa; 24.08.1982; JAA; 1 ex. - Pagazuri, (Zigoitia), Álava; 22.06.2016; FH; 1 ex. - Bóveda, (Valdegobia), Álava; 26.10.2016; FH: 1 ex. Primera cita de Álava.

Género Megalinus Mulsant \& Rey, 1877

Megalinus glabratus (Gravenhorst, 1802). - Ondiz, (Leioa); 10.05.1992; FH; 1 ex. Primera cita de Bizkaia.

Género Nudobius Thomson, 1860

Nudobius collaris (Erichson, 1839). - Ulia, (Donostia), Gipuzkoa; 15.07.1982; FG; 1 ex. Primera cita de Gipuzkoa. 
Género Stenistoderus Jaquelin du Val, 1856

Stenistoderus nothus (Erichson, 1839). - Agiturri, (Kuartango), Álava; 18.07.2015; FH; 1 ex. Primera cita de Álava.

Género Xantholinus Dejean, 1821

Subgénero Purrolinus Coiffait, 1962

Xantholinus tricolor (Fabricius, 1787). - Artikutza, (Goizueta), Navarra; 07.08.1995; LMM; 4 ex. Primera cita de Navarra.

Subgénero Xantholinus Dejean, 1821

Xantholinus linearis (Olivier, 1795). - Gorbea, (Zuia), Álava; 18.05.2009; FH; 1 ex. Primera cita de Álava.

Xantholinus longiventris (Heer, 1839). - Ondiz, (Leioa), Bizkaia; 08.04.1994; FH; 1 ex. Primera cita de Bizkaia.

\section{Discusión}

El conjunto alcanza 102 especies diferentes con bastante desproporción numérica de ejemplares entre ellas. Las altitudes medias de los lugares de captura oscilan entre los 500 y 1500m con excepción de las zona costera de San Sebastian / Donostia por haber sido la residencia de la mayoría de los socios. Estudiando el conjunto de los Coleoptera de la Sociedad de Ciencias Aranzadi se deduce que la familia Staphylinidae es cuantitativamente pequeña si se compara con otras familias como Scarabaeidae, Carabidae, etc. La posible causa sería que las especies se recolectaron subsidiariamente a otras familias y de ahí la característica de que un $23 \%$ del conjunto sea de hábito coprófilo. Tomando como referencia inicial el Catálogo Iberobalear de los Staphylinidae de Outerelo y Gamarra (2005, 2007, 2008 y 2009) con sus sucesivas actualizaciones (Gamarra \& Outerelo, 2010, 2012, 2014) se añaden en el listado las primeras citas de las provincias indicadas (muchas especies son bastante vulgares) siempre considerando las posibilidades de corrección a causa de citas en otros trabajos dispersos. Es de destacar la presencia de algunas especies como las que indicamos a continuación:

Olophrum piceum (Gyllenhal, 1810). Especie europea escasa.

Dinothenarus (s.st.) pubescens (De Geer, 1774). Especie euroturánica de distribución extensa en Europa pero localmente escasa, (Fig. 1).

Paraphloeostiba gayndahensis (Mac Leay, 1873). Especie invasora de origen australiano y asiático extendiéndose por los huertos familiares sobre hortalizas. 
Philonthus (Onychophilonthus) marginatus (O.Müller, 1764). Especie llamativa por la coloración de su pronoto de distribución euroasiática y hábito coprófilo situado a niveles subalpinos y altimontanos, (Fig. 2).

Quedius (Microsaurus) mesomelinus (Marsham, 1802). Especie cosmopolita y abundante interesante por ocupar lugares umbríos sobre todo en las cuevas del País Vasco. Fuertemente atraído a la trampas de caída cebadas con materia orgánica animal.



Fig. 1.- Dinothenarus pubescens (De Geer, 1774)

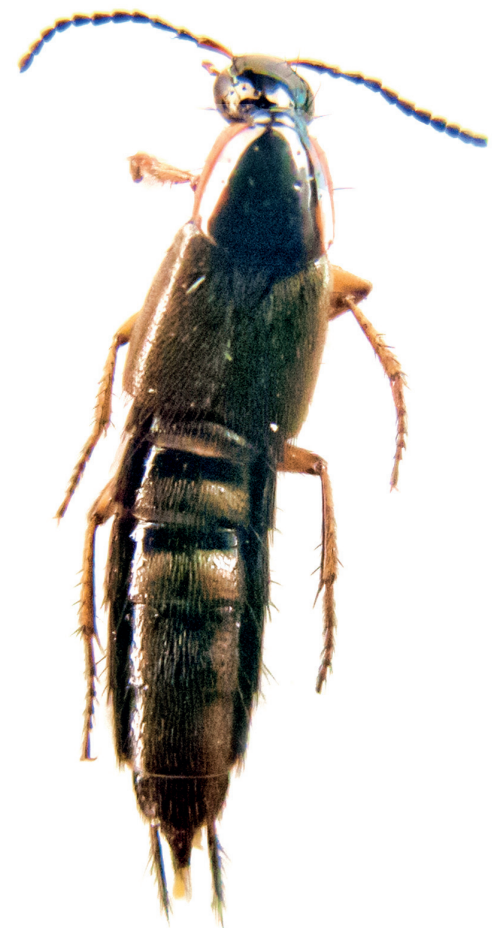

Fig. 2.- Philonthus marginatus (Müller, 1764) 


\section{Bibliografía}

- Anichtchenko, A. 2006. Estudio del orden Coleoptera en las marismas de Txingudi. Disponible en: www.osakidetza.euskadi.eus/r85-ckserv01/es/contenidos/memoria/pdf.

-Assing,V. 2010. A revision of Achenium. (Coleoptera: Staphylinidae: Paederinae). Nova Supplementa Entomologica 21: 1 - 190.

- Díaz-Martín, B., Saloña-Bordas, M.I. 2015. Arthropods of forensic interest associated to pig carcasses in Aiako Harria Natural Park (Basque Country, Northern Spain). Cienc. Forense 12: 207-228.

- Coiffait, H. 1972-1984. Coleopteres Staphylinidae de la Region Palearctique Occidentale. Vol. 1-V. CNRS. Toulouse.

- Gamarra, P., Outerelo, R. 2005. Catálogo iberobalear de los Aleocharinae (Coleoptera: Staphylinidae). Bol. Soc. Entomol. Aragonesa 37: 1-81.

- Gamarra, P., Outerelo, R. 2007. Catálogo iberobalear de los Paederinae (Coleoptera: Staphylinidae). Bol. Soc. Entomol. Aragonesa 40: 1-37.

- Gamarra, P., Outerelo, R. 2008a. Catálogo iberobalear de los Staphylininae (Coleoptera: Staphylinidae). Bol. Soc. Entomol. Aragonesa 42: 197-251.

- Gamarra, P., Outerelo, R. 2008b. Catálogo iberobalear de los Omaliinae (Coleoptera: Staphylinidae). Bol. Soc. Entomol. Aragonesa 43: 211-231.

- Gamarra, P., Outerelo, R. 2008c. Catálogo iberobalear de los Oxytelinae (Coleoptera: Staphylinidae). Bol. Soc. Entomol. Aragonesa 43: 233-254.

- Gamarra, P., Outerelo, R. 2009c. Catálogo iberobalear de los Tachyporinae (Coleoptera: Staphylinidae). Bol. Soc. Entomol Aragonesa 44: 183-200.

- Gamarra, P., Outerelo, R. 2009d. Catálogo iberobalear de los Steninae (Coleoptera: Staphylinidae). Bol. Soc. Entomol. Aragonesa 45: 181-200.

- Gamarra, P., Outerelo, R. 2010. Primera actualización del catálogo Iberobalear de los Staphylininae. Disponible en: http://bba.bioucm.es/cont/docs/RO 14. pdf .

- Gamarra, P., Outerelo, R. 2012. Actualización del catálogo Iberobalear de los Omaliinae Disponible en: http://bba.bioucm.es/cont/docs/RO 18. pdf.

- Gamarra, P., Outerelo, R. 2014. Primera actualización al catálogo de los Oxytelinae de la Península Ibérica. Disponible en: http://bba.bioucm.es/cont/docs/RO 27. pdf

- Gamarra, P., Outerelo, R. 2014. Segunda actualización del catálogo Iberobalear de los Aleocharinae. Disponible en: http://bba.bioucm.es/cont/docs/RO 9. pdf

- Gamarra, P., Outerelo, R., 2014. Segunda actualización del catálogo Iberobalear de los Paederinae. Disponible en: http://bba.bioucm.es/cont/docs/RO 2. pdf

- Lompe, A. 2002. Die Käfer Europas. Ein Bestimmungswerk in Internet. Familie Staphylinidae. Disponible en: www.coleo-net.de/coleo/index.htm

- Lott, D.A., Anderson, R. 2011. The Staphylinidae (rove beetles) of Britain and Ireland. Parts 7\&8: Oxyporinae, Steninae, Euasthetinae, Pseudopsinae, Pederinae, Staphylininae. Handbooks for the Identification of British Insects. Vol. 12, Part 7. Royal Entomological Society. 
- Outerelo, R., Gamarra, P., San Martín, A.F., Recalde, J.I. 2016. Estudio de los estafilínidos de un viejo robledal submediterráneo de Navarra (norte de España), (Coleoptera: Staphylinidae). Bol. Real Soc. Esp. Hist. Nat. Secc. Biol. 110: 33-46.

- Outerelo, R., Gamarra, P., San Martín, A.F., Recalde, J.I. 2016. Staphylinidae (Coleoptera) del Parque Natural del Señorío de Bertiz (Navarra, norte de España). Arquivos Entomolóxicos 15: 321 336. Disponible en: www.aegaweb.com/arquivos_entomoloxicos

- Pagola-Carte, S., Zabalegi, I., Hernando, C. 2011. Altxor Entomologiko Aiako Harria Parke naturalean. Sustrai 77: 52-55.

- Portevin, G. 1929. Histoire Naturelle des Coléoptères de France. Tome I. Poliphaga: Staphylinoidea. Paul Lechevalier editeur, Paris.

- Saloña, M.I., Moraza. M.L., Carles-Tolrá, M., Iraola, V., Bahillo, P., Yélamos, T., Outerelo, R., Alcaraz, R. 2010. Searching the Soil: Forensic Importance of Edaphic Fauna After the Removal of a Corpse. J. Forensic Sci. Disponible en: http://bba.bioucm.es/cont/docs/220.pdf

- Tronquet, M. 2014. Catalogue Iconographique des Coléoptères des Pyrénées-Orientales. Volume I. Staphylinidae. Association Roussillonnaise d'Entomologie.

- Vorst, O. 2013. On some Gipuzkoan Coleoptera, including several species new to the Iberian Peninsula. Heteropterus, Rev. Entomol. 13: 147-173.

- Zanetti, A. 2008. Synonymies in the European Omaliinae, with notes on distribution. (Coleoptera: Staphylinidae). Linzer biol. Beitrag. 40(1): 979-992. 